\title{
The disruption of mitochondrial axonal transport is an early event in neuroinflammation
}

\author{
Oihana Errea', Beatriz Moreno', Alba Gonzalez-Franquesa, Pablo M. Garcia-Roves ${ }^{2,3,4}$ and Pablo Villoslada ${ }^{1,5^{*}}$ (D)
}

\begin{abstract}
Background: In brain inflammatory diseases, axonal damage is one of the most critical steps in the cascade that leads to permanent disability. Thus, identifying the initial events triggered by inflammation or oxidative stress that provoke axonal damage is critical for the development of neuroprotective therapies. Energy depletion due to mitochondrial dysfunction has been postulated as an important step in the damage of axons. This prompted us to study the effects of acute inflammation and oxidative stress on the morphology, transport, and function of mitochondria in axons.

Methods: Mouse cerebellar slice cultures were challenged with either lipopolysaccharide (LPS) or hydrogen peroxide $\left(\mathrm{H}_{2} \mathrm{O}_{2}\right)$ ex vivo for $24 \mathrm{~h}$. Axonal mitochondrial morphology was evaluated by transmission electron microscopy (TEM) and mitochondrial transportation by time-lapse imaging. In addition, mitochondrial function in the cerebellar slice cultures was analyzed through high-resolution respirometry assays and quantification of adenosine triphosphate (ATP) production.

Results: Both conditions promoted an increase in the size and complexity of axonal mitochondria evident in electron microscopy images, suggesting a compensatory response. Such compensation was reflected at the tissue level as increased respiratory activity of complexes I and IV and as a transient increase in ATP production in response to acute inflammation. Notably, time-lapse microscopy indicated that mitochondrial transport (mean velocity) was severely impaired in axons, increasing the proportion of stationary mitochondria in axons after LPS challenge. Indeed, the two challenges used produced different effects: inflammation mostly reducing retrograde transport and oxidative stress slightly enhancing retrograde transportation.

Conclusions: Neuroinflammation acutely impairs axonal mitochondrial transportation, which would promote an inappropriate delivery of energy throughout axons and, by this way, contribute to axonal damage. Thus, preserving axonal mitochondrial transport might represent a promising avenue to exploit as a therapeutic target for neuroprotection in brain inflammatory diseases like multiple sclerosis.
\end{abstract}

Keywords: Mitochondria, Axonal transport, Axonal damage, Inflammation, Oxidative stress, Multiple sclerosis

\section{Background}

Axonal damage is the main factor contributing to the disability associated with many brain diseases caused by inflammation, oxidative stress, neurodegeneration, or ischemia. For example, in the case of an inflammatory disease like multiple sclerosis (MS), it is known that axonal damage occurs from the outset of the disease [1-3] and

\footnotetext{
* Correspondence: pvilloslada@clinic.ub.es

'Center of Neuroimmunology, Institut d'Investigacions Biomèdiques August Pi Sunyer (IDIBAPS), Cellex Building, Laboratory 3A, Casanova 145, 08036 Barcelona, Spain

${ }^{5}$ University of California, San Francisco, USA

Full list of author information is available at the end of the article
}

that it is the main contributor to long-term disability [4-6]. Axonal damage may be triggered by diverse mechanisms, progressing along the different phases of the disease. In the early inflammatory stages, axonal damage is mainly provoked by the inflammatory cascade, microglia activation, oxidative stress, and mitochondrial dysfunction [7-9]. By contrast, in more chronic stages, energy demands increase as a result of the loss of trophic support provided by myelin and the consequent redistribution of sodium channels, increasing the calcium inside axons and leading to axonal fragmentation due to the unsatisfied energy demands [10-12]. 
Disease progression in a mouse model of MS (experimental autoimmune encephalomyelitis (EAE)) is associated with reversible modifications to axons and other events that provoke axonal transection [13]. However, the timing of each event and the contribution of each process to transient and definitive axonal damage are not well understood. The identification of the early events leading to axonal damage after insult is critical to develop neuroprotective therapies that may decrease permanent axonal damage and disability in brain diseases such as MS.

Mitochondrial dysfunction seems to be one of the critical steps in axonal damage [14-16], and indeed, mitochondrial complex IV dysfunction has been detected in the axons, oligodendrocytes, and hypertrophied astrocytes of hypoxia-like pattern III MS lesions [17]. A microarray postmortem analysis of the non-myelinated motor cortex from MS patients revealed a reduction in the expression of 26 mitochondrial respiratory chain subunits and in the activity of complexes I, III, and IV [18]. Moreover, morphological changes to the mitochondria were detected inside axons undergoing focal axonal degeneration, as a step prior to the morphological changes and axonal fragmentation in EAE [13]. More recently, impaired axonal transport has been observed in EAE animals prior to the structural alterations to axons, their cargo, or microtubules. These deficits in transport began to revert within a few days of the peak of EAE, and they were reversed by anti-inflammatory and antioxidative interventions [19].

In this study, we set out to assess the contribution of mitochondrial abnormalities induced by inflammation or oxidative stress to axonal damage. By identifying the early processes involved in axonal damage, we should be able to promote the development of neuroprotective therapies targeting these processes. We performed in vivo imaging and molecular analyses of ex vivo murine cerebellar slice cultures challenged by agents that provoke inflammation (lipopolysaccharide (LPS)) or oxidative stress $\left(\mathrm{H}_{2} \mathrm{O}_{2}\right)$. We focused on these two types of insult because they are particularly relevant to the acute inflammatory damage in MS relapses and, to some extent, they contribute to other diseases like brain trauma, ischemia, or neurodegeneration. Our results point to the disruption of mitochondrial axonal transport as an early pathogenic mechanism that triggers axonal damage during acute neuroinflammation.

\section{Materials and methods}

\section{Cerebellar slice cultures and stimulations}

Organotypic slice cultures were prepared from the cerebellum of 7-day-old C57BL/6J mice (Janvier Labs), obtaining $300 \mu \mathrm{m}$ sagittal slices with a Mcllwain Tissue Chopper (Mickle Laboratory). Three or four slices were placed on Cell Culture Inserts (Millipore) in six-well plates and cultured at $37{ }^{\circ} \mathrm{C}$ in an atmosphere of $5 \%$
$\mathrm{CO}_{2}$ with $1 \mathrm{ml}$ of organotypic slice medium: $50 \%$ basal medium containing Earle's salt (Gibco, Invitrogen), $25 \%$ Hank's buffered salt solution (Gibco, Invitrogen), $25 \%$ inactivated horse serum (Gibco, Invitrogen), $5 \mathrm{mg} / \mathrm{ml}$ glucose (Sigma-Aldrich), $0.25 \mathrm{mM} \mathrm{L-glutamine} \mathrm{(Gibco,}$ Invitrogen), $25 \mu \mathrm{g} / \mathrm{ml}$ penicillin/streptomycin (Gibco, Invitrogen), and $1 \times$ anti-mycotic (Gibco, Invitrogen). The medium was replaced every $2-3$ days, and to reduce microglial activation and to permit axonal myelination, the slices were maintained for 7 days in vitro (DIV) before adding any stimulus. After 7 DIV, the slices were treated with Escherichia coli LPS $(15 \mu \mathrm{g} / \mathrm{ml}$; SigmaAldrich) or $\mathrm{H}_{2} \mathrm{O}_{2}(500 \mu \mathrm{M}$; Sigma-Aldrich) for different times $(3,6,24,48,72$, and $96 \mathrm{~h})$ as described elsewhere [20]. Time course studies revealed that the earliest and most significant differences were observed $24 \mathrm{~h}$ after the challenge, and as such, this time point was used thereafter. The dose of $\mathrm{H}_{2} \mathrm{O}_{2}$ used previously for $24 \mathrm{~h}$ stimulations $\left(1 \mathrm{mM} \mathrm{H}_{2} \mathrm{O}_{2}\right)$ [20] was reduced to $500 \mu \mathrm{M}$ to ensure better axonal survival at longer times of stimulation (data not shown). Untreated control slices were incubated for identical periods as the treated cultures: up to 8 DIV for $24 \mathrm{~h}$ stimulations and up to 11 DIV for experiments at different time points.

All animal handling was carried out in accordance with the European Council Directive (2010/63/EU) and the Spanish regulations for the procurement and care of experimental animals (1201 RD/2005, October 10). All the study protocols were approved by the Ethical Committee on Animal Research of the University of Barcelona.

\section{Transmission electron microscopy (TEM)}

Cerebellar slices were fixed in $2 \%$ paraformaldehyde and $2.5 \%$ glutaraldehyde in $0.1 \mathrm{M}$ phosphate buffer $(\mathrm{PB})$ for $24 \mathrm{~h}$ at $4{ }^{\circ} \mathrm{C}$, and after washing for $12 \mathrm{~h}$ with several changes of $0.1 \mathrm{M} \mathrm{PB}$, the slices were post-fixed with $2 \%$ osmium tetroxide in $0.1 \mathrm{M} \mathrm{PB}$ for $1 \mathrm{~h}$ at $4{ }^{\circ} \mathrm{C}$. The tissue was dehydrated in increasing concentrations of methanol and flat embedded in Epoxy-embedding medium. Longitudinal sections were obtained along the original sagittal plane of the organotypic cultures, and only white matter areas were selected for ultramicrotomy (Fig. 1a). Ultra-thin sections were stained with a solution of $1 \%$ uranyl acetate and lead citrate (all reagents from SigmaAldrich), and the sections were observed on a JEOL 1010 transmission electron microscope (JEOL, USA). Images of axonal mitochondria were acquired with a MegaView III digital camera (Olympus) and analyzed with ImageJ Software. Two different regions of interest were selected by hand for the analysis, the external mitochondrial perimeter, and the internal cristae perimeter (Fig. 1b). The mitochondrial morphology parameters are described in Table 1 [21, 22]. 


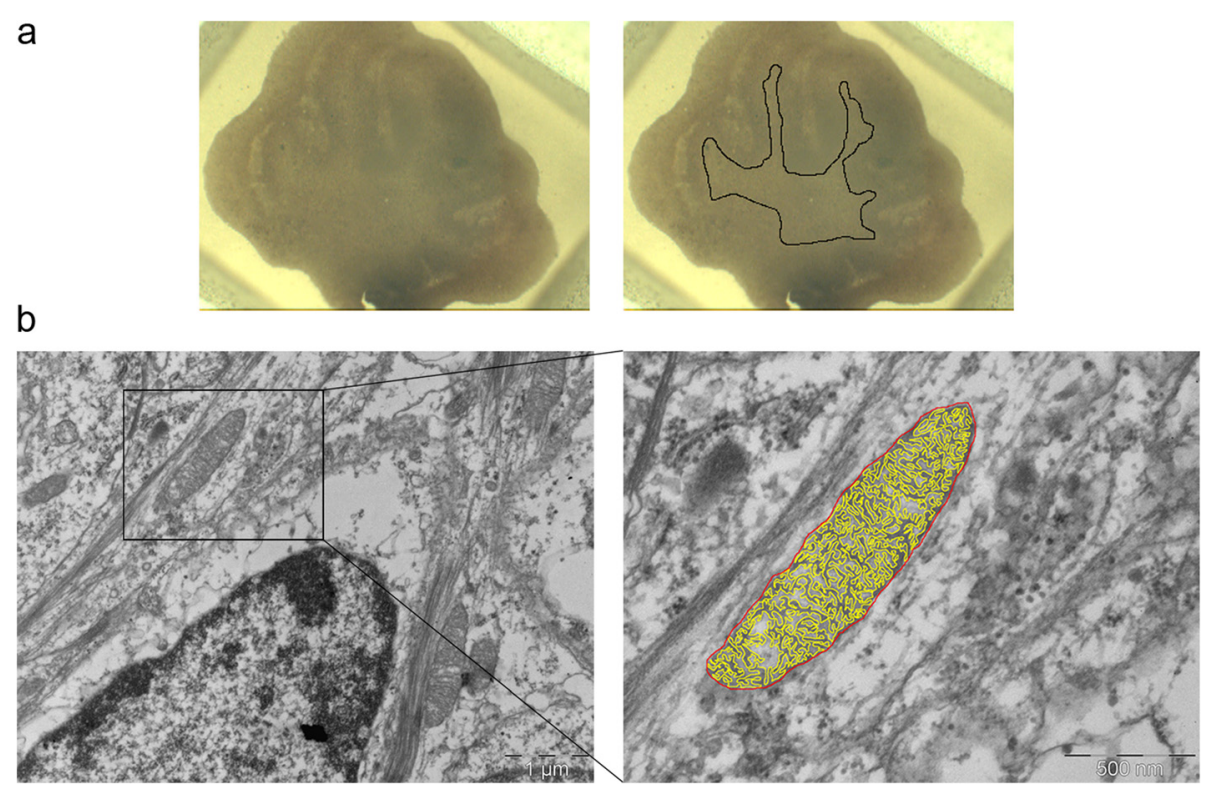

Fig. 1 Analysis of axonal mitochondrial morphology by transmission electron microscopy in cerebellar slice cultures. a Images of an organotypic cerebellar slice culture embedded in an Epoxy resin block (left image) and the white matter area selected for ultramicrotomy (area inside the black line, right image). b Segmentation method to study axonal mitochondrial morphology. Left image shows a section of cerebellar white matter with a longitudinally cut axon and a mitochondrion inside. The external mitochondrial perimeter (red line, right image) and the internal cristae perimeter (yellow line, right image) were drawn manually using ImageJ. Scale bars, 1 um left image and $500 \mathrm{~nm}$ right image

\section{Preparation of lentiviral particles, microinjection into cerebellar slice cultures, and time-lapse imaging}

A lentivirus containing mitochondrial-targeted DsRed2 was generously donated by Dr. Bruce D. Trapp (Department of Neurosciences, Cleveland Clinic Lerner Research Institute) [23]. The plasmid was amplified in competent DH5 $\alpha$ E. coli (Invitrogen) transformed following the manufacturer's instructions, and $293 \mathrm{~T}$ cells were transfected with the construct and packaging plasmids using Lipofectamine 2000 (Invitrogen) according to the manufacturer's protocol. Viral supernatants were collected $48 \mathrm{~h}$ after the transfection, centrifuged at $3000 \mathrm{~g}$ for $15 \mathrm{~min}$ and filtered through $0.45 \mu \mathrm{m}$ polyvinylidene difluoride (PVDF) filters (Millipore) to eliminate the cell debris. Subsequently, the viral supernatants were centrifuged at 110,000 $g$ for $3 \mathrm{~h}$ at $4{ }^{\circ} \mathrm{C}$ to concentrate the viral particles, which were resuspended in a $1 / 100$ volume of PBS, aliquoted, and frozen at $-80^{\circ} \mathrm{C}$ until use.

Two hours after plating the cerebellar slices, the lentiviral particles were injected into the Purkinje cell layer using a microinjector (InjectMan, Eppendorf) and a micromanipulator (FemtoJet, Eppendorf) connected to a spinning disk confocal microscope (Revolution XDi System, Andor Technology) following the protocol described elsewhere [24]. Borosilicate capillaries with an external diameter of $2 \mathrm{~mm}$ (Warner Instruments) and stretched to a 5-15 $\mu \mathrm{m}$ diameter at the tip were used to perform three to four injections per slice.

After 7 DIV and the corresponding stimulation, Purkinje cell axons with DsRed2-positive mitochondria were visualized as reported previously [23, 25]. Cell culture inserts with the slices attached were transferred to glass-

Table 1 Morphological parameters of mitochondria analyzed by transmission electron microscopy

\begin{tabular}{ll}
\hline Parameter & Meaning \\
\hline Mitochondrial area & Total area of each mitochondrion \\
External perimeter & Mitochondrial external perimeter \\
Feret's diameter & Longest distance between any two points of the mitochondrial external perimeter \\
Circularity & Two-dimensional sphericity index. A value of 1 corresponds to a perfect sphere. Circ $=\frac{4 \pi \times \text { Area }}{(\text { Perimeter })^{2}}$ \\
Roundness & Two-dimensional sphericity index. A value of 1 corresponds to a perfect sphere. Round $=\frac{4 \times \text { Area }}{\pi(\text { minoraxis })^{2}}$ \\
Aspect ratio (AR) & Ratio between the major and the minor axis of a mitochondrion. AR $=\frac{\text { Major Axis }}{\text { Minor Axis }}$ \\
Cristae perimeter/external & Ratio between the mitochondrial cristae perimeter and the external perimeter. Reflects the complexity of \\
perimeter & mitochondrial cristae
\end{tabular}


bottom dishes (MatTek Corporation) with $1 \mathrm{ml}$ of culture medium. Images were acquired for $20 \mathrm{~min}$ at $512 \times 512$ pixel resolution on an inverted spinning disk confocal microscope equipped with a cage incubator and an EMCCD camera (Revolution XDi System, Andor Technology). The resultant recordings were analyzed using Imaris software (Bitplane) that automatically generates tracks corresponding to moving objects (Fig. 2).
Stationary mitochondria were identified as objects without any displacement during the recording time.

\section{High-resolution respirometry}

High-resolution respirometry assays were performed on an Oxygraph-2k system (Oroboros Instruments, Austria), and for each measurement, 15 cerebellar slices were
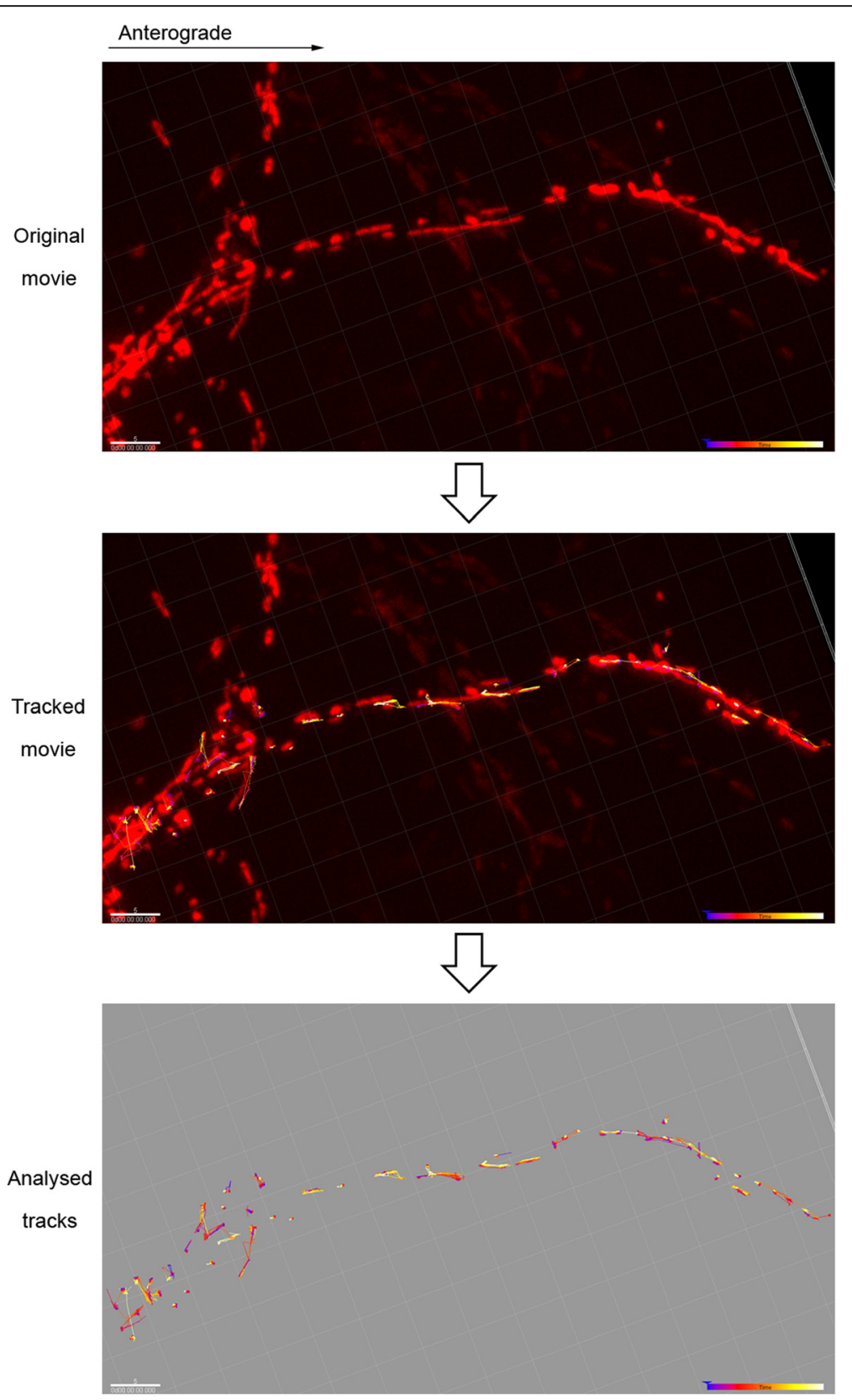

Fig. 2 In vivo imaging of axonal mitochondrial transport in cerebellar slice cultures. (Top) Image stack extracted from an original recording showing an individual axon containing DsRed2-positive mitochondria. (Middle) Motile mitochondria were identified and tracked automatically using the autoregressive motion algorithm in the ImarisTrack package (Imaris software, Bitplane). (Bottom) The resulting tracks are time-color coded, and the software automatically calculates several parameters for each track, such as track area mean $\left(\mu \mathrm{m}^{2}\right)$ or track speed mean $(\mu \mathrm{m} / \mathrm{s})$. Scale bars $5 \mu \mathrm{m}$, time bars 20 min 
homogenized in $150 \mu \mathrm{l}$ of MIR05 mitochondrial respiration buffer at $\mathrm{pH} 7.1$ (0.5 mM EGTA, $3 \mathrm{mM} \mathrm{MgCl}_{2}$, $60 \mathrm{mM}$ K-lactobionate, $20 \mathrm{mM}$ taurine, $10 \mathrm{mM} \mathrm{KH}_{2} \mathrm{PO}_{4}$, $20 \mathrm{mM}$ HEPES, $110 \mathrm{mM}$ sucrose, $1 \mathrm{~g} / \mathrm{l}$ BSA) [26]. Homogenized tissue $(100 \mu \mathrm{l})$ was added to $2 \mathrm{ml}$ of MIR05 in the different chambers of the respirometer. DatLab (Oxygraph-2k-associated software) provides respiratory values for the previously defined states by calculating the negative derivative of the oxygen concentration with respect to time.

The SUIT (substrate-uncoupler-inhibitor-titration) protocol used was as follows (the concentrations indicated are saturating and the final concentrations in the chambers): malate ( $2 \mathrm{mM}$; Sigma) and glutamate $(10 \mathrm{mM}$; Sigma) to measure the LEAK uncoupled respiration state (due to proton leakage and the circuit of electrons and cations that is not dependent on ATP synthase activity); ADP (5 mM; Calbiochem) $+\mathrm{MgCl}_{2}$ (0.6 mols per each ADP mol) for the measurement of reduced nicotinamide adenine dinucleotide (NADH)-dependent respiration through complex I; the OXPHOS state, where the gradient of protons pumped into the mitochondrial intermembrane space is partially utilized by ATP synthase for oxidative phosphorylation, coupled respiration, and that is also partially dissipated due to proton leakage or uncoupled respiration; cytochrome C (10 $\mu \mathrm{M}$; Sigma) was added to the chambers to verify if the mitochondrial membrane had been damaged during sample preparation (no significant increase in oxygen consumption was observed); succinate (10 mM; Sigma) to measure reduced flavin adenine dinucleotide $\left(\mathrm{FADH}_{2}\right)$-dependent complex II respiration together with complex I respiration in the OXPHOS CI + CII state; oligomycin $(2 \mu \mathrm{g} / \mathrm{ml}$; Sigma $)$ to detect endogenous uncoupled respiration from ATP synthesis through complexes I and II substrates by inhibiting ATP synthase; carbonyl cyanide-p-trifluoromethoxyphenylhydrazone (FCCP) $(0.5+0.5 \mu \mathrm{M}$; Sigma) until reaching the maximum respiration to quantify the maximum capacity of the electron transport system by nonphysiological uncoupling of the internal mitochondrial membrane (ETS CI + CII state); rotenone $(0.5 \mu \mathrm{M}$, Sigma) to inhibit complex I and thereby study the maximum respiration corresponding only to complex II (ETS CII); and antimycin A (2.5 $\mu \mathrm{M}$; Sigma) to inhibit complex III and define the ROX (residual oxygen consumption) state that is related to non-mitochondrial respiration, in order to subtract this value from the previous values. Finally, ascorbate ( $2 \mathrm{mM}$; Sigma) and $N, N, N^{\prime}, N^{\prime}$-tetramethyl- $p$-phenylenediamine dihydrochloride (TMPD) (0.5 mM; Sigma) were added to analyze complex IV respiration. Ascorbate avoids TMPD self-oxidation and maintains it in its reduced state. Throughout the SUIT protocol, each reagent was added when the previous respiratory signal was stable for at least 2-3 min. After respirometry assays, the total protein in each sample was measured by the Bradford method (Sigma) to normalize the respiration values.

\section{Western blots}

Four cerebellar slices were plated in each well, and after 7 DIV and the corresponding stimulations, the slices were collected in $100 \mu \mathrm{l}$ of RIPA buffer (Sigma) supplemented with protease inhibitor cocktail (Sigma). The slices were homogenized, centrifuged for $5 \mathrm{~min}$ at $12,000 \mathrm{rpm}$ and $4{ }^{\circ} \mathrm{C}$, and the supernatant was recovered and the total protein was measured by the Bradford method (Sigma).

To detect SUO (70 kDa Fp subunit of the succinate ubiquinone oxidoreductase or complex II) and COX IV (subunit IV of the cytochrome c oxidase or complex IV), $40 \mu \mathrm{g}$ of total protein was resolved on $4-12 \%$ Criterion XT Bis-Tris gels (Bio-Rad) in MES buffer (Bio-Rad), more appropriate to separate low molecular weight proteins. Proteins were transferred to PVDF membranes (GE Healthcare), previously activated for $1 \mathrm{~min}$ with $100 \%$ methanol. The membranes were then blocked for $1 \mathrm{~h}$ at room temperature with TBS $(0.05 \%$ Tween, $5 \%$ dry milk) and incubated overnight at $4{ }^{\circ} \mathrm{C}$ with the primary antibodies diluted in blocking solution: mouse anti-SUO, 1:1000 (Molecular Probes) or mouse antiCOX IV, 1:1000 (Molecular Probes). After several washes with TBS-Tween $0.05 \%$, the membranes were incubated for $1 \mathrm{~h}$ at room temperature with the secondary horseradish peroxidase (HRP)-conjugated antimouse IgG antibody (GE Healthcare) diluted in blocking solution $(1: 10,000)$. Protein loading was assessed and normalized to the signal from a HRP-conjugated anti- $\beta$ actin antibody (Sigma) diluted 1:25,000 in blocking solution and incubated for $20 \mathrm{~min}$ at room temperature.

\section{ATP measurements}

ATP production in the organotypic cerebellar slice cultures was measured using a bioluminescent assay (ATP Determination Kit, Molecular Probes) based on the ATP requirements of a recombinant firefly luciferase to produce light (emission maximum $\sim 560 \mathrm{~nm}$ at $\mathrm{pH} 7.8$ ). The experiments were carried out according to the manufacturer's instructions. Briefly, the slices were collected from each well in $50 \mu \mathrm{l}$ of deionized water and homogenized. Samples were transferred to a dark 96-well plate (Nunc), and a standard curve of different ATP concentrations was established from 0.001 to $1 \mathrm{nM}$. Finally, the plate was read in a luminometer (GloMax, Promega) using the following parameters: delay time $1 \mathrm{~s}$, integration time $10 \mathrm{~s}$, and injected reaction volume $100 \mu \mathrm{l}$.

\section{Statistical analysis}

All statistical analyses were performed using SPSS 15.0 (IBM) and GraphPad Prism 6 (GraphPad Software) 
software, presenting the data as the mean \pm SEM. The negative control and the LPS and $\mathrm{H}_{2} \mathrm{O}_{2}$ (24 h treatment) data were analyzed by one-way ANOVA and with a Dunnett's multiple comparisons post hoc test when a Gaussian distribution was followed, or with a KruskalWallis test and Dunn's multiple comparisons post hoc test or a Mann-Whitney $U$ test plus Bonferroni's correction for post hoc multiple comparisons when samples didn't follow a Gaussian distribution. For the experiments in which treatments were added at different time points, the data were analyzed by two-way ANOVA applying a Dunnett's multiple comparisons post hoc test. Frequency analysis was performed with the chi-squared test. $P$ values are presented as ${ }^{*} p<0.05,{ }^{* * *} p<0.01$, and $* * * * 0.001$.

\section{Results}

Inflammation and oxidative stress provoke an increase in the size and cristae complexity of axonal mitochondria Changes to the mitochondria in axons undergoing inflammatory degeneration have previously been demonstrated in an animal model of MS and EAE [13]. Hence, we set out to characterize these morphological changes in axonal mitochondria in response to neuroinflammation (triggered by LPS-induced microglia activation) and oxidative stress (triggered by $\mathrm{H}_{2} \mathrm{O}_{2}$ ), in an ex vivo model using cerebellar slice cultures [20]. In such model, we have found that LPS also induces oxidative stress by inducing iNOS expression and increasing levels of reactive oxygen species [20]. Accordingly, longitudinally cut axons were identified in the white matter of cerebellar slices and the morphological parameters of the axonal mitochondria were analyzed in TEM images $24 \mathrm{~h}$ after the insult (Fig. 1 and Table 1).

Both these challenges increased the mitochondrial area compared to the untreated control (LPS vs control: $p=0.029 ; \mathrm{H}_{2} \mathrm{O}_{2}$ vs. control: $p=0.001$; Fig. 3a). Moreover, the external perimeter of the mitochondria increased following the neuroinflammatory challenge ( $p=0.037$ compared to the control) but not oxidative stress $(p=0.135$; Fig. 3b). No differences were observed when considering Feret's diameter ( $p=0.069$, Kruskal-Wallis test; Fig. 3c).

The shape of axonal mitochondria was analyzed using three different descriptors: circularity, roundness, and aspect ratio (Table 1). We did not find significant changes between the mitochondria in control and LPS-stimulated slices (Fig. 3d-f), although the axonal mitochondria in the slices exposed to LPS had a tendency to elongate (negative control, $\quad \mathrm{AR}=2.691 \pm 0.191 ; \quad \mathrm{LPS}, \quad \mathrm{AR}=3.092 \pm 0.249$; Fig. 3f). By contrast, axonal mitochondria were more rounded following oxidative stress than in the control slices (circularity $p=0.004$, roundness $p=0.033$, AR $p=0.033$; Fig. 3-f).
Finally, as changes to the organization of the mitochondrial internal membrane regulate mitochondrial respiratory function $[27,28]$, the complexity of axonal mitochondrial cristae was analyzed, reflected by the ratio of the mitochondrial cristae perimeter and the external perimeter. This ratio increased beyond that of the controls after both challenges (LPS vs. control: $p<0.0001$; and $\mathrm{H}_{2} \mathrm{O}_{2}$ vs. control: $p<0.0001$; Fig. $3 \mathrm{~g}, \mathrm{~h}$ ). Thus, the acute response of axonal mitochondria to the inflammatory and oxidative insults in vitro involves an increase in their size and cristae complexity, suggesting compensatory changes in respiratory function.

\section{Changes in the distribution of stationary and motile axonal mitochondria after damage}

Mitochondrial transport in axons is extremely important to correctly locate the mitochondria at sites of higher energetic demand, such as the nodes of Ranvier and synaptic terminals, as well as for mitochondrial renewal when the mitochondria that have lost their respiratory efficacy are destroyed [29-31]. Altered mitochondrial transport may compromise axonal survival even though mitochondrial function per se might not be disrupted. Therefore, we analyzed the acute changes in mitochondrial transport along axons after the inflammatory challenge and oxidative stress by in vivo imaging of the cerebellar slices in culture. Neuronal mitochondria were labeled by microinjection of lentiviral particles containing a mitochondrial-targeted DsRed2 sequence close to Purkinje cell somas, a lentivirus that preferentially infects neurons and not glial cells [23]. Purkinje cell axons with positive DsRed 2 mitochondria were visualized $24 \mathrm{~h}$ after LPS or $\mathrm{H}_{2} \mathrm{O}_{2}$ challenge.

We first quantified the total mitochondrial area in each axon registered and normalized this to the axon length to assess whether the total axonal mitochondrial content was affected by either challenge (LPS or $\mathrm{H}_{2} \mathrm{O}_{2}$ ). Axonal mitochondrial density was not affected by $24 \mathrm{~h}$ of neuroinflammation or oxidative stress ( $p=0.198$, Kruskal-Wallis test; Fig. 4a), indicating that the mitochondria were not significantly depleted from axons due to the damage induced.

Two different populations of the mitochondria can be distinguished in axons in terms of their transport: stationary mitochondria and motile mitochondria [32, 33]. In healthy axons, stationary mitochondria are located at the nodes of Ranvier where there is high energy demand required for the transmission of nerve impulses. Motile mitochondria move in an anterograde (towards the distal axon) or retrograde direction (towards the soma) from one site of stationary mitochondria to the next. Thus, the distribution of the different mitochondrial populations was analyzed in the three experimental conditions (Fig. 4b). In the control tissue, $31.3 \%$ of the mitochondria 
a

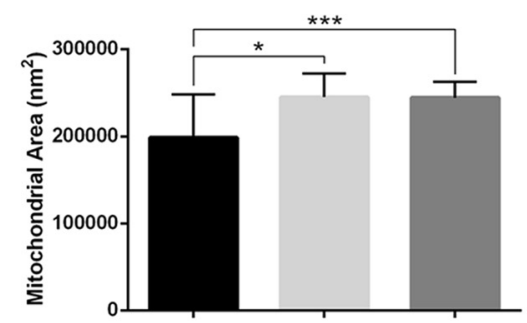

b

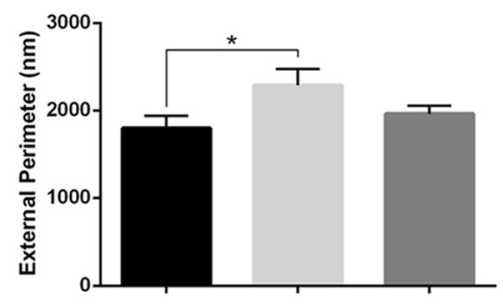

C

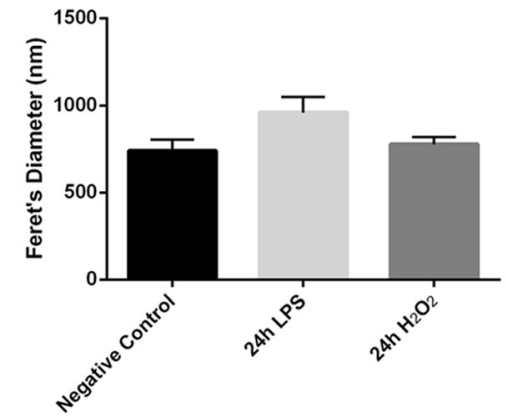

d

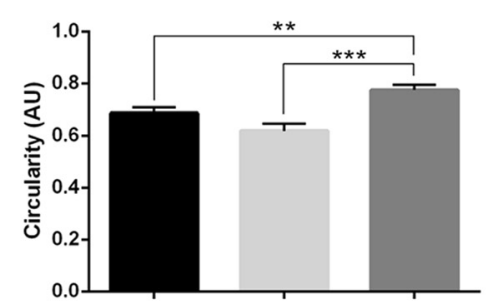

e

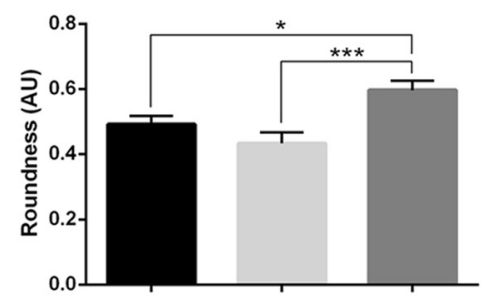

f

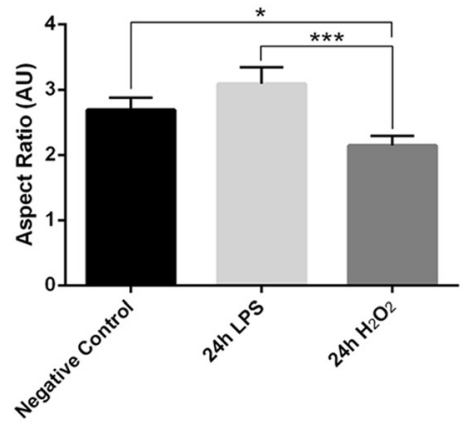

g

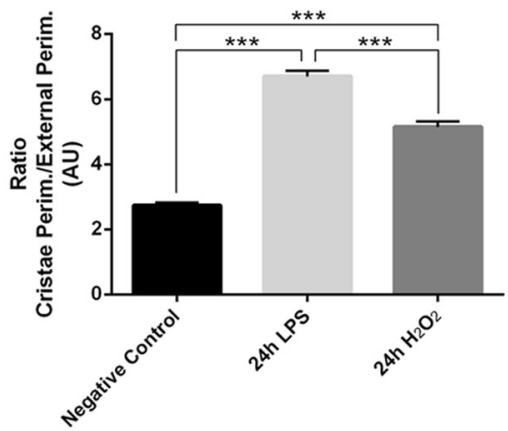

$\mathrm{h}$

Negative Control

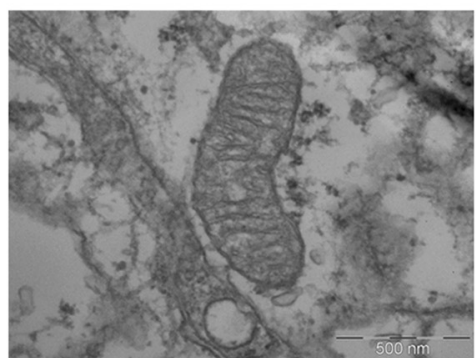

24h LPS

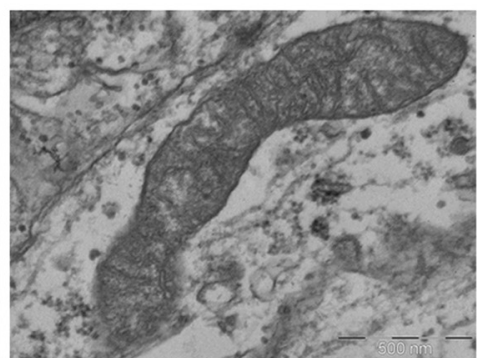

24h $\mathrm{H}_{2} \mathrm{O}_{2}$

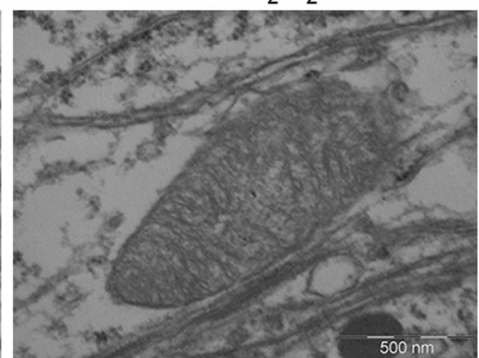

Fig. 3 (See legend on next page.) 
(See figure on previous page.)

Fig. 3 Effects of neuroinflammation and oxidative stress on axonal mitochondrial morphology. a-c Mitochondrial size parameters: a area, $\mathbf{b}$ external perimeter, and $\mathbf{c}$ Feret's diameter. a Axonal mitochondria increase their area in conditions of neuroinflammation and oxidative stress. $\mathbf{b}$ However, the external perimeter only increases significantly during neuroinflammation, c while Feret's diameter also has a tendency to increase in the neuroinflammatory condition. $\mathbf{d}$-f Mitochondrial shape descriptors: $\mathbf{d}$ circularity, e roundness, and $\mathbf{f}$ aspect ratio. Axonal mitochondria are more rounded following oxidative stress than in unstimulated and LPS-stimulated cultures. $\mathbf{g}$ The ratio between the internal cristae perimeter and the external perimeter indicates that both neuroinflammation and oxidative stress induce an increase in axonal mitochondrial cristae complexity. a-g $n=73$ control mitochondria, 50 LPS-challenged mitochondria and $75 \mathrm{H}_{2} \mathrm{O}_{2}$-challenged mitochondria from two different experiments: Kruskal-Wallis test and Dunn's multiple comparisons post hoc test. Mean \pm SEM. (AU arbitrary units); ${ }^{*} p<0.05$, ${ }^{* *} p<0.01,{ }^{* * *} p<0.001$. h Representative TEM images of axonal mitochondria from unstimulated, LPS-, and $\mathrm{H}_{2} \mathrm{O}_{2}$-stimulated organotypic cerebellar slice cultures. Scale bars $500 \mathrm{~nm}$

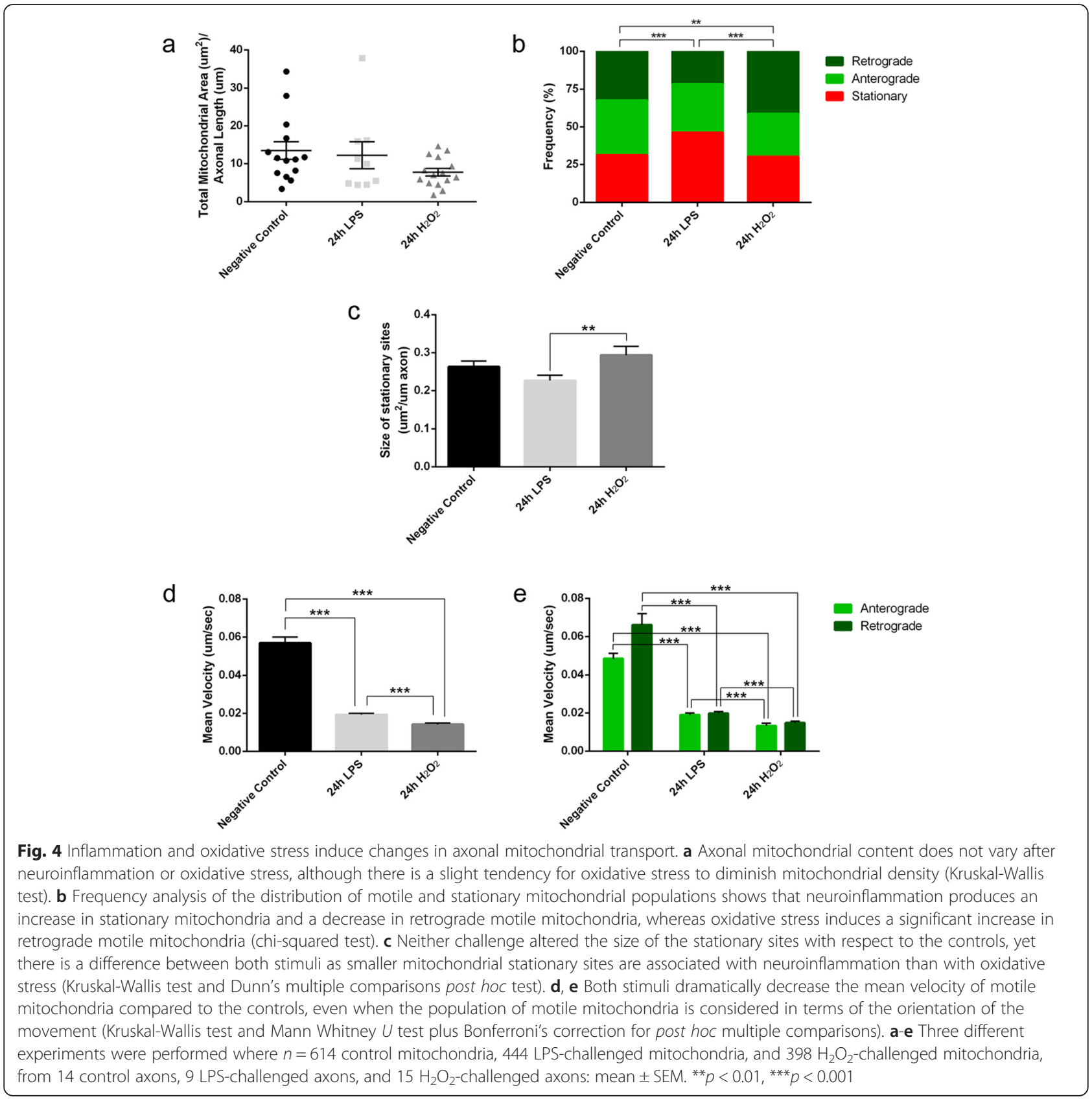


were stationary and $68.7 \%$ were motile $(36.1 \%$ anterograde and $32.6 \%$ retrograde), whereas neuroinflammation increased the amount of stationary mitochondria to $46.2 \%$, decreasing the amount of retrograde motile mitochondria to $21.6 \%$ (32.2 \% anterograde; $p<0.0001$ with respect to the controls, chi-squared test). By contrast, oxidative stress did not affect the percentage of stationary mitochondria (30.2\%), although the percentage of retrograde motile mitochondria increased significantly to $41.2 \%$ (28.6 \% anterograde; $p=0.010$ with respect to the controls, chi-squared test).

\section{Axonal mitochondrial transport is severely disrupted by neuroinflammation and oxidative stress}

Stationary mitochondria were identified as objects that were not displaced during the recording, although it is more accurate to name these as stationary mitochondrial sites since the resolution of axon recording did not allow us to distinguish if the fluorescent signal came from one or more mitochondria. The area occupied by objects that were not displaced within the stationary site was calculated and normalized to the axon's length. There was a tendency for the size of the stationary sites to diminish following neuroinflammation but not oxidative stress (LPS vs control, $p=0.088 ; \mathrm{H}_{2} \mathrm{O}_{2}$ vs. control, $p=0.719$; Fig. 4c). Moreover, there was a significant difference in the size of stationary sites when the effect of the two stimuli was compared $(p=0.0059)$.

Motile mitochondria were identified as objects with a displacement velocity greater than zero at any moment in the recordings. Axonal mitochondria described diverse trajectories: maintaining the same direction, switching from anterograde to retrograde displacement and vice versa, moving at the beginning and then stopping, etc. For the mitochondria that go through a stationary site, it is impossible to know whether the same mitochondrion enters and leaves. Thus, in such cases, the trajectories were separated as belonging to different mitochondria. The parameter selected to assess the motile behavior of axonal mitochondria was the mean velocity [34], which decreased dramatically with respect to the controls after each challenge (LPS vs. control, $p<0.0001 ; \mathrm{H}_{2} \mathrm{O}_{2}$ vs. control, $p<0.001$; Fig. 4d). Even when differentiating between the anterograde and retrograde trajectories, the differences between these insults (LPS or $\mathrm{H}_{2} \mathrm{O}_{2}$ ) and the controls persisted (Fig. 4e). However, retrograde transport was affected distinctly and while inflammation severely decreased retrograde mitochondrial transport, it was enhanced by oxidative stress (Fig. 4b). In summary, neuroinflammation and oxidative stress severely affect the speed of axonal mitochondrial transport, whereby inflammatory damage preferentially impaired retrograde transport.

\section{Increased respiratory capacity in response to} neuroinflammation and oxidative stress

Mitochondrial function in the cerebellar slice cultures was analyzed by using high-resolution respirometry. Since the cerebellar tissue is homogenized for these assays, the signal obtained comes from all the cell types in the tissue, including glial cells, and thus these results cannot be related directly to the observations in single axonal mitochondria described above. The two main processes related to mitochondrial respiration were analyzed: the transport of electrons obtained from NADH and $\mathrm{FADH}_{2}$ oxidation through the electron transport system (formed by complexes I to IV), which generates a proton gradient in the intermembrane space (through the respirometry assays and the analysis of different respiratory states; Table 2), and the oxidative phosphorylation, or ATP synthesis from ADP and inorganic phosphorus ( $\mathrm{Pi}$ ) catalyzed by ATP synthase (complex V), which uses the electrochemical gradient generated by electron transport as a driving force (measured as the ATP produced in the cultures).

The initial LEAK state of uncoupled respiration and NADH-dependent complex I respiration (CI) increased in conditions of neuroinflammation (LPS) compared to the control $(p<0.0001$ for LEAK and $p<0.0001$ for CI, one-way ANOVA and Dunnett's multiple comparisons post hoc test; Fig. 5a). However, complex II was not affected, as the ETS II state (after the inhibition of complex I) was not different from the controls $(p=0.107)$. Therefore, the differences in the CI + CII $(p=0.048)$, uncoupling ( $p=0.023)$, and ETS I + II $(p=0.018)$ states observed following LPS stimulation were mainly due to enhanced complex I respiration (Fig. 5a). During oxidative stress $\left(\mathrm{H}_{2} \mathrm{O}_{2}\right)$, only the initial LEAK state of uncoupled respiration $(p=0.002)$ and NADH-dependent complex I respiration $(\mathrm{CI})(p=0.041)$ were enhanced relative to the controls (Fig. 5a).

The order in which complex IV substrates (ascorbate and TMPD) were added in the SUIT protocol was changed and optimized from $\mathrm{H}_{2} \mathrm{O}_{2}$ stimulations to LPS stimulations. Therefore, to be able to compare those measurements, LPS and $\mathrm{H}_{2} \mathrm{O}_{2}$ complex IV (CIV) respiratory values were normalized to their corresponding negative control respiratory values. Complex IV respiration increased after LPS stimulation $(p=0.009)$ but not after $\mathrm{H}_{2} \mathrm{O}_{2}$ stimulation $(p=0.990)$ (Fig. $\left.5 \mathrm{~b}\right)$.

To determine whether the increased respiration of some mitochondrial complexes was triggered by the increase of their enzymatic/respiratory capacity or by increased protein expression, two mitochondrial complexes were analyzed by Western blots (Fig. 5c): the succinate ubiquinone oxidoreductase or complex II (SUO) and the cytochrome c oxidase or complex IV (COX IV). The expression of SUO and COX IV did not change significantly following 
Table 2 Respiratory states analyzed in the SUIT protocol designed for high-resolution respirometry assays

\begin{tabular}{|c|c|}
\hline Respiratory state & Meaning \\
\hline LEAK & $\begin{array}{l}\text { Respiration state uncoupled from ATP synthase activity, due to proton leakage and the circuit of electrons } \\
\text { initiated after the addition of malate and glutamate in the absence of ADP }\end{array}$ \\
\hline Complex I (Cl) & $\begin{array}{l}\text { Respiration state of NADH-dependent complex I after the addition of ADP, in which the proton gradient of the } \\
\text { intermembrane space is partially used by ATP synthase for oxidative phosphorylation (coupled respiration) and } \\
\text { partially dissipated due to proton leakage (uncoupled respiration) }\end{array}$ \\
\hline Complexes I and II (Cl + CII) & $\begin{array}{l}\text { Measurement of } \mathrm{FADH}_{2} \text {-dependent complex || respiration together with complex I respiration after the } \\
\text { addition of succinate }\end{array}$ \\
\hline Uncoupling & $\begin{array}{l}\text { Endogenous respiration uncoupled from ATP synthesis through complex I and II substrates after the addition } \\
\text { of oligomycin (ATP synthase inhibitor) }\end{array}$ \\
\hline $\begin{array}{l}\text { Electron transfer system capacity I + } \\
\text { II (ETS I + II) }\end{array}$ & $\begin{array}{l}\text { Measurement of the electron transfer system's maximum capacity through the non-physiological uncoupling of } \\
\text { the internal mitochondrial membrane after the addition of FCCP (an ionophore or uncoupling agent that } \\
\text { dissipates } \mathrm{H}^{+} \text {) }\end{array}$ \\
\hline $\begin{array}{l}\text { Electron transfer system capacity } \| \\
\text { (ETS II) }\end{array}$ & Maximum respiration that only corresponds to complex II after the addition of rotenone (complex I inhibitor) \\
\hline Complex IV (CIV) & $\begin{array}{l}\text { Respiration state of complex IV after complex III inhibition by antimycin A, and the addition of ascorbate } \\
\text { and TMPD }\end{array}$ \\
\hline
\end{tabular}

Explanation of all the mitochondrial respiratory states included in the SUIT protocol designed for high-resolution respirometry assays. The respiratory states were measured in a continuous manner (without breaks between enzymatic reactions), and in this way, the substrates from the first respiratory states are available for the following states

either challenge (SUO, $p=0.254$ one-way ANOVA; COX, $p=0.118$ one-way ANOVA; Fig. $5 \mathrm{~d}$, e). In summary, the acute response of the cerebellar tissue to either inflammation or oxidative stress involves an increase in respiratory capacity, mainly through complexes I and IV, although we cannot define which cell type was responsible for this process.

\section{Neuroinflammation induces a parallel increase in ATP production and respiratory activity in stressed cerebellar slice cultures}

To determine whether the increase in the respiratory capacity of some mitochondrial complexes in the cerebellar slice cultures induced an increment in ATP production, we quantified ATP in a bioluminescent assay. Accordingly, ATP production appeared to increase after LPS stimulation $(p=0.0007)$, although this increase was transient and peaked at $24 \mathrm{~h}$, it was no longer evident after $96 \mathrm{~h}$ (Fig. 5f). By contrast, no significant increase in the production of ATP was provoked by oxidative stress.

\section{Discussion}

In this study, the acute effects of neuroinflammation and oxidative stress on axonal mitochondria have been analyzed, particularly with respect to their morphology and transport. Likewise, the functional consequences of these insults were assessed in terms of respiratory function and ATP production. We found that in response to either challenge, axonal mitochondria respond by increasing their size and cristae complexity, which may suggest an effort to increase energy production following acute damage. Mitochondria control their morphology through fusion and fission processes. Thus, the increase in axonal mitochondrial size suggests that the mitochondria might fuse to exchange mitochondrial components between damaged and healthy axonal mitochondria, thereby maintaining a functional population. Moreover, axonal mitochondria undergo an internal reorganization. The inner membrane structure is related to the mitochondrion's metabolic state [28], and in more active respiratory states with high ATP concentrations, the mitochondria adopt a "condensed" morphology with a large number of cristae [27]. These results suggest that the mitochondria respond to the increased metabolic needs of axons during acute inflammation and oxidative stress.

The morphological alterations to the mitochondria observed during neuroinflammation occurred in parallel to the enhanced respiratory function of some mitochondrial complexes and the production of ATP by the tissue. Although we cannot determine which cell types were responsible for the increase in respiration and ATP, we predict glial cells will have a strong influence, such as microglia or astrocytes. However, the morphological changes in axonal mitochondria suggest that such changes contribute to the enhanced respiratory capacity and energy production soon after damage (within $24 \mathrm{~h}$ ). We did not assess longer times because we were interested in early events, although several studies indicate that energy production decreases in the central nervous system (CNS) of animals suffering long-term inflammatory diseases such as EAE [35, 36].

Mitochondrial electron transport is the main biological process leading to ROS generation [37, 38], and higher metabolic rates correlate with higher levels of oxidative stress [39]. Indeed, the transient increase of ATP production (peaked after $24 \mathrm{~h}$ of LPS stimulation) correlates 
a

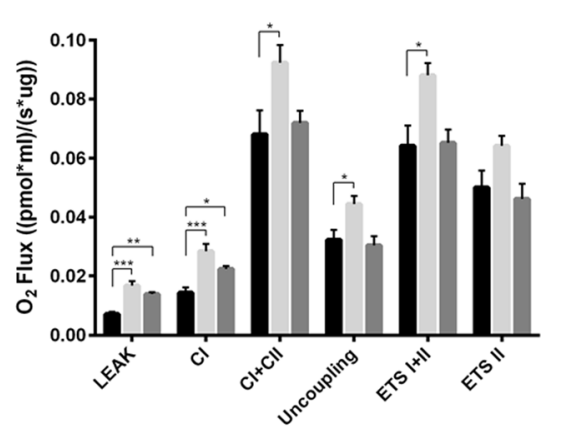

b

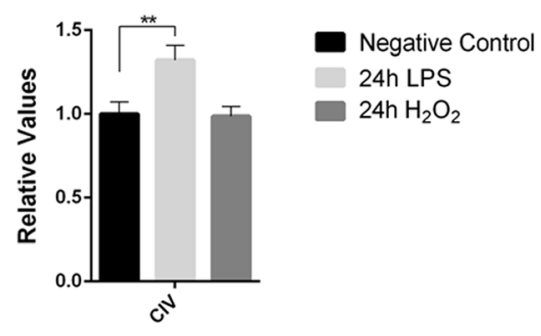

SUO (CII)
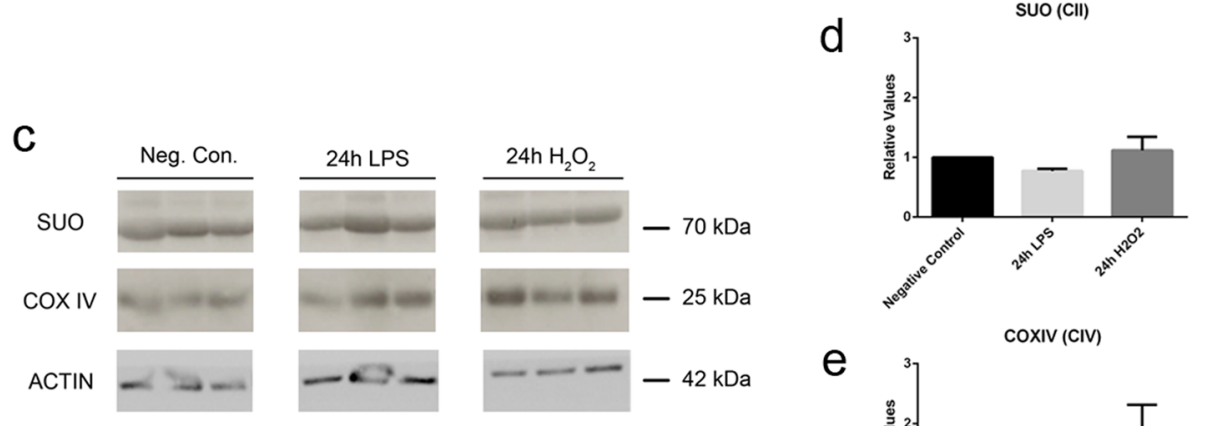

e

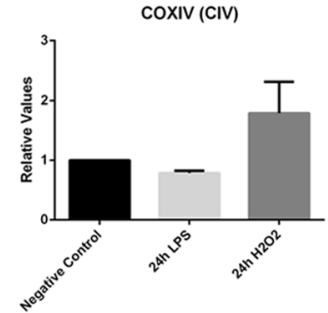

f

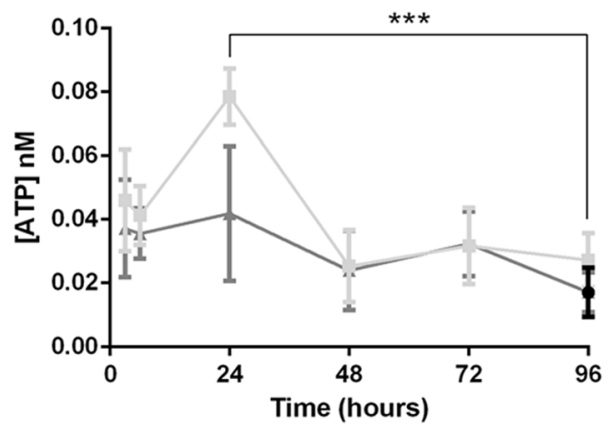

Fig. 5 Effects of neuroinflammation and oxidative stress on mitochondrial function in cerebellar slice cultures. a Respiratory values of the cerebellar slice cultures show that neuroinflammation (LPS) produces an increase of LEAK, $\mathrm{Cl}, \mathrm{Cl}+\mathrm{Cll}$, uncoupling, and ETS I + II respiratory states, while oxidative stress $\left(\mathrm{H}_{2} \mathrm{O}_{2}\right)$ causes an increase of LEAK and $\mathrm{Cl}$ respiratory states $\left(n=13\right.$ controls, 9 LPS and $\left.5 \mathrm{H}_{2} \mathrm{O}_{2}\right)$. One-way ANOVA and Dunnett's multiple comparisons post hoc test for the respiratory states in which samples have a Gaussian distribution, Kruskal-Wallis test and Mann Whitney $U$ test plus Bonferroni's correction post hoc multiple comparisons for the respiratory states in which samples do not have a Gaussian distribution (mean \pm SEM; ${ }^{*} p<0.05,{ }^{* *} p<0.01$, $\left.{ }^{* * *} p<0.001\right)$. b Respiratory values for mitochondrial complex IV were obtained in different experiments for $\mathrm{LPS}$ and $\mathrm{H}_{2} \mathrm{O}_{2}$ stimulations. Therefore, CIV values were normalized with the respiratory values of the negative control in each experiment. The resulting relative values show that only LPS produces an increase of CIV respiratory state (controls $n=12$, LPS $n=9 ; \mathrm{H}_{2} \mathrm{O}_{2} n=7$ ). One-way ANOVA and Dunnett's multiple comparisons post hoc test (mean \pm SEM; $\left.{ }^{* *} p<0.01\right)$. $\mathrm{c}$ Western blot showing the expression of two subunits from two different mitochondrial electron transport chain complexes in the organotypic cerebellar slice cultures: the 70-kDa Fp subunit of the succinate ubiquinone oxidoreductase or complex II (SUO); and subunit IV of the cytochrome c oxidase or complex IV (COX IV). d, e Quantification of the Western blot shows no significant differences in SUO or COX IV expression ( $n=3$, one-way ANOVA; mean \pm SEM). $\mathbf{f}$ The LPS challenge significantly augments ATP production in the organotypic cerebellar slice cultures at $24 \mathrm{~h}(n=3$, two-way ANOVA and Dunnett's multiple comparisons post hoc test; mean \pm SEM; ${ }^{* * *} p<0.001$ )

with an increased oxidative stress in the tissue confirmed by an increment of iNOS expression and ROS levels [20]. In that study [20], we quantified iNOS expression (by Western blot) and ROS production (using a fluorescent assay with $\mathrm{H}_{2}$ DCFDA) at different times after LPS challenge $(0-96 \mathrm{~h})$. iNOS protein levels 
increased at 12-24 $\mathrm{h}$ after LPS challenge and decreased subsequently, and ROS levels peaked at $12 \mathrm{~h}$ and remained increased up to the study end point. Active mitochondria producing high levels of ATP in high metabolic states also produce more ROS, and probably those high ROS levels damage the mitochondria and impede the production of high ATP levels in longer time points rather than $24 \mathrm{~h}$.

We also found that mitochondrial transportation is the earliest dysfunction of axonal mitochondria in response to neuroinflammation and oxidative stress. A strong decrease in the mean transport velocity was provoked by both insults and both directions, anterograde and retrograde.
This is in accordance with the impaired axonal transport seen previously, even before axonal demyelination or structural alterations were observed [19]. This decrease in the mean velocity of axonal mitochondrial transport is critical because axons are very long and they have very active structures that require significant amounts of energy, such as synapses, nodes of Ranvier, active growth cones, or axonal branches $[40,41,29]$. If mitochondrial transport is impaired in axons, there would be a decrease in the energy (ATP) available at these sites of strong demand. In the nodes of Ranvier, such deficits would impair the activity of the $\mathrm{Na}^{+} / \mathrm{K}^{+}$ATPase, which would in turn promote the reversion of axonal membrane $\mathrm{Na}^{+} / \mathrm{Ca}^{2+}$ transporters

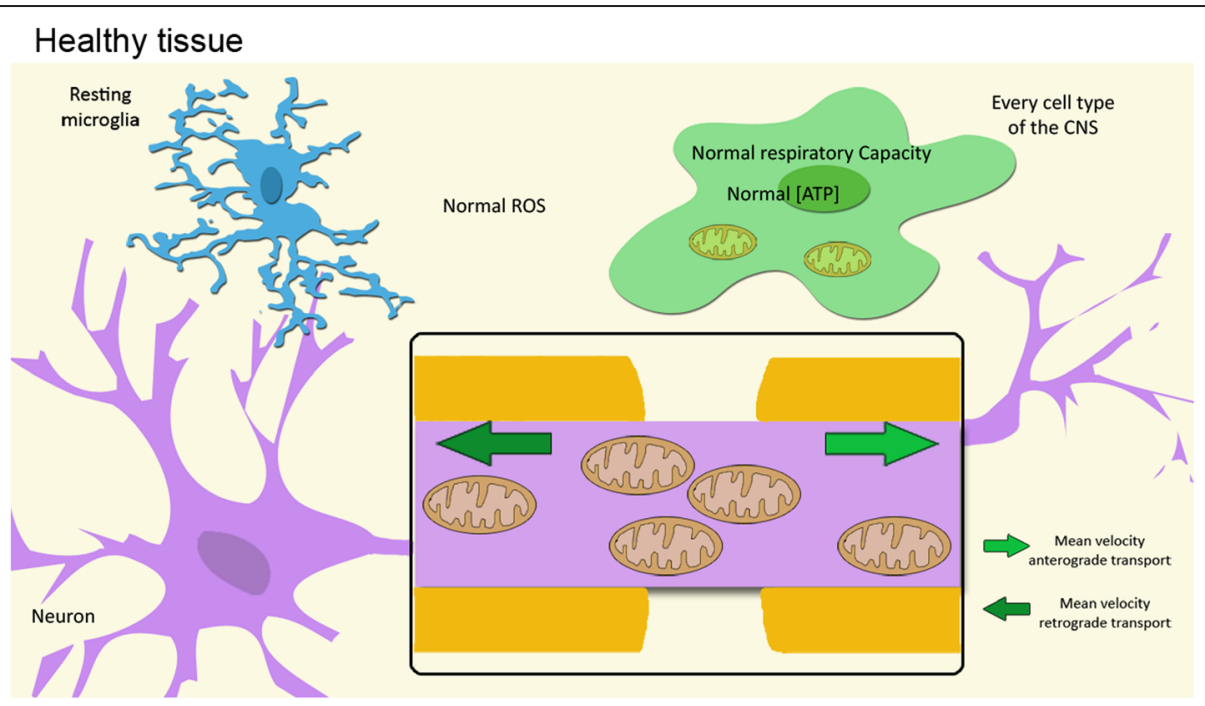

Acute inflammation

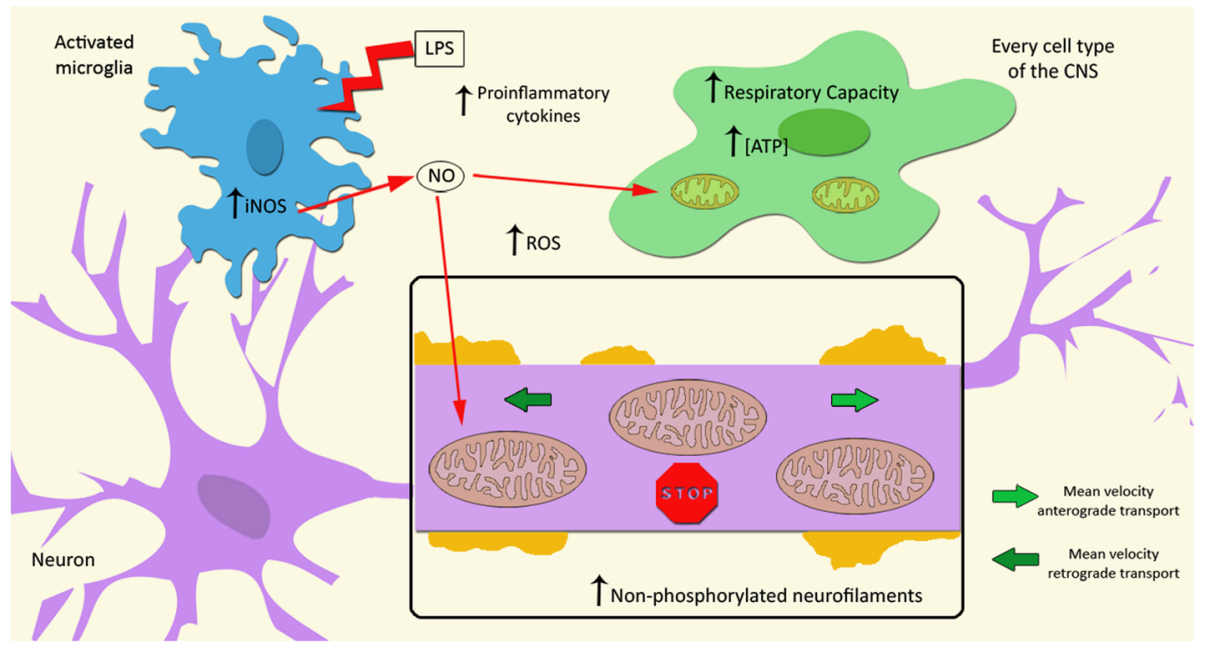

Fig. 6 Mitochondrial response to acute neuroinflammation. The model of the mitochondrial response to an acute neuroinflammatory environment proposed shows that the mitochondria respond at the tissue level by increasing their respiratory capacity and ATP production, whereas at the axonal level mitochondria respond by increasing their size and cristae complexity, although their transport is disrupted. Mitochondrial paralysis occurs early, before axonal damage is irreversible. Therefore, disruption of axonal mitochondrial transport (mainly retrograde) is a critical mechanism underlying mitochondrial dysfunction during neuroinflammation. Our results, and previous studies in this model [20], suggest that preserving axonal mitochondrial transport could represent a neuroprotective therapy for acute CNS inflammation 
and provoke an increase in $\mathrm{Ca}^{2+}$ levels in the axoplasm that could trigger several degenerative processes $[42,16]$.

Interestingly, we found that each insult has a specific effect on the mitochondria. The mitochondria adopt a more rounded shape in response to oxidative stress, like the mitochondria of swollen axons in the EAE model [13]. Furthermore, while ATP production did not increase, the respiratory capacity of the LEAK state and CI increased less than during inflammation. Both observations indicate that oxidative stress is more directly damaging the mitochondria than inflammation. In terms of axonal mitochondrial transport, although transportation speed was severely reduced by both insults, neuroinflammation augments the number of stationary mitochondria and dampens retrograde mitochondrial motility, whereas oxidative stress increases the amount of retrograde motile mitochondria. The fact that retrograde mitochondrial transport increases after oxidative damage suggests that early damaged mitochondria are transported to the soma for elimination, yet additional mitochondria are not transported anterogradely because they may became not functional. By contrast, the increase in the number of stationary mitochondria during neuroinflammation is consistent with the increased density of stationary mitochondria in EAE axons seen previously [19]. Given that the size of the stationary sites is smaller following neuroinflammation than following oxidative stress, we propose that the mitochondria that were motile before the insult (motile mitochondria are smaller than stationary mitochondria [43]) stop and accumulate all along the axon during inflammation.

Regarding the differential mechanisms of axonal damage associated with CNS inflammation, we previously described that LPS triggers microglia activation in this ex vivo model of cerebellar slice cultures, which secrete proinflammatory cytokines and produce reactive oxygen species [20]. In this model, axonal damage seems to be highly dependent on oxidative stress, whereas pro-inflammatory cytokines contribute significantly to demyelination. The differential effects of such challenges in this study suggest that pro-inflammatory mediators have a significant effect on mitochondrial axonal transport, either through directly damaging axons or as an indirect effect of acute demyelination. Most importantly, at the time, we observed mitochondrial morphological changes and the disruption of axonal mitochondrial transport; axons were already damaged to some extent [20]. Both stimuli significantly increased the amount of dephosphorylated neurofilaments, indicative of the onset of axonal damage. Previous studies [2] have shown dephosphorylated neurofilaments being present in acute lesions from MS patients. Therefore, all the mitochondrial changes that we observed appear to occur at the initial stages of axonal damage either during neuroinflammation or oxidative stress.
It remains unclear what might cause the dramatic decrease in axonal mitochondrial transport velocity, either in neuroinflammation or oxidative stress. Considering that both anterograde and retrograde transport is affected, the different motor proteins in charge of such transport (kinesins for anterograde and dyneins for retrograde transport) may not be the primary cause. Therefore, we hypothesize that inflammatory and oxidative processes somehow alter the attachment of motor/ adaptor/mitochondrial complexes to microtubules. Identifying the specific sites of damage in the axon cargo system will be critical to develop therapeutic strategies that prevent permanent axonal damage and disability.

Our study focused on the acute response to inflammatory and oxidative stress in order to identify the earliest process involved in axonal degeneration. Although this is important to identify future neuroprotective strategies, our results cannot explain how the CNS responds to chronic damage. Our data was obtained in an ex vivo model in order to benefit from high-resolution imaging techniques, and they are in agreement with previous observations made in the EAE animal model [19]. We found that the main complexes that react to neuroinflammation, complexes I and IV, are the same as those preferentially altered in brain lesions of MS patients [17, 35, 44]. Therefore, and based on current evidence, we hypothesize that after acute inflammation such as MS relapses, the mitochondria compensate for the extra energy demands by increasing their size, cristae complexity, and the respiratory capacity of complexes I and IV. However, due to impairment in axonal mitochondrial transport, axons will suffer energy delivery depletion at sites of high-energy demand such as the nodes of Ranvier. This energy delivery depletion, in addition to other factors triggered by inflammation, will promote axonal damage (Fig. 6).

\section{Conclusions}

The results presented here show that after acute inflammation and oxidative stress in the CNS, axonal mitochondria respond to the augmented energy demands by increasing their size and cristae complexity. However, axonal mitochondrial transport is rapidly and severely impaired, preventing the delivery of energy along axons and to specific sites of high-energy demands. Such unattended energy demands may trigger several degenerative processes, leading to axonal damage and subsequent transection. Therefore, addressing these axonal mitochondrial transport deficits could revert the progression of axonal damage to irreversible axonal degeneration.

\section{Abbreviations}

ADP: adenosin diphosphate; ANOVA: analysis of variance; AR: aspect ratio; ATP: adenosin triphosphate; Cl: complex I; Cll: complex II; CIV: complex IV; CNS: central nervous system; COX IV: sytochrome c oxidase subunit IV; 
DIV: days in vitro; EAE: experimental autoimmune encephalomyelitis; ETS: electron transfer system; $\mathrm{FADH}_{2}$ : reduced flavin adenine dinucleotide; FCCP: carbonyl cyanide- $p$-trifluoromethoxyphenylhydrazone; $\mathrm{H}_{2} \mathrm{O}_{2}$ : hydrogen peroxide; LEAK: leak oxygen flux; LPS: lipopolysaccharide; MS: multiple sclerosis; NADH: reduced nicotinamide adenine dinucleotide; OXPHOS: oxidative phosphorylation; ROX: residual oxygen consumption SUIT: substrate-uncoupler-inhibitor-titration; SUO: succinate ubiquinone oxidoreductase; TEM: transmission electron microscopy; TMPD: $N, N, N^{\prime}, N$ '-tetramethyl-p-phenylenediamine dihydrochloride.

\section{Competing interests}

The authors declare that they have no competing interests.

\section{Authors' contributions}

$\mathrm{OE}, \mathrm{BM}$, and PV designed the study. OE and BM performed the TEM, time-lapse imaging, and ATP measurements experiments. OE, AG-F, and PMG-R carried out the high-resolution respirometry assays and Western blots. $\mathrm{OE}, \mathrm{BM}, \mathrm{AG}-\mathrm{F}$, and PMG-R analyzed data. AG-F and PMG-R provided substantial contributions to the design and interpretation of the data. OE and PV wrote the manuscript. All authors read and approved the final manuscript.

\section{Acknowledgements}

We thank Nagore Escala and Begoña Fernandez for their technical support. This work was supported by the Instituto de Salud Carlos III (FIS: PI12/01823) to PV and Ministerio de Ciencia e Innovación (BFU2011-24679) to PMG-R. PMG-R is a recipient of a Ramon y Cajal contract (RYC-2009-05158) from Ministerio de Ciencia e Innovación.

\section{Author details}

'Center of Neuroimmunology, Institut d'Investigacions Biomèdiques August Pi Sunyer (IDIBAPS), Cellex Building, Laboratory 3A, Casanova 145, 08036 Barcelona, Spain. ${ }^{2}$ Diabetes and Obesity Research Laboratory, Institut d'Investigacions Biomèdiques August Pi Sunyer (IDIBAPS), 08036 Barcelona, Spain. ${ }^{3}$ Spanish Biomedical Research Center in Diabetes and associated disorders (CIBERDEM), University of Barcelona, 08907 Barcelona, Spain. ${ }^{4}$ Department of Physiological Sciences II, University of Barcelona, 08907 Barcelona, Spain. ${ }^{5}$ University of California, San Francisco, USA.

\section{Received: 21 April 2015 Accepted: 16 August 2015}

\section{Published online: 28 August 2015}

\section{References}

1. Ferguson B, Matyszak MK, Esiri MM, Perry VH. Axonal damage in acute multiple sclerosis lesions. Brain. 1997;120(Pt 3):393-9.

2. Trapp BD, Peterson J, Ransohoff RM, Rudick R, Mork S, Bo L. Axonal transection in the lesions of multiple sclerosis. N Engl J Med. 1998:338:278-85

3. Filippi M, Bozzali M, Rovaris M, Gonen O, Kesavadas C, Ghezzi A, et al. Evidence for widespread axonal damage at the earliest clinical stage of multiple sclerosis. Brain. 2003;126:433-7.

4. Trapp BD, Ransohoff R, Rudick R. Axonal pathology in multiple sclerosis: relationship to neurologic disability. Curr Opin Neurol. 1999;12:295-302.

5. Kornek B, Storch MK, Weissert R, Wallstroem E, Stefferl A, Olsson T, et al. Multiple sclerosis and chronic autoimmune encephalomyelitis: a comparative quantitative study of axonal injury in active, inactive, and remyelinated lesions. Am J Pathol. 2000;157:267-76.

6. Kuhlmann T, Lingfeld G, Bitsch A, Schuchardt J, Bruck W. Acute axonal damage in multiple sclerosis is most extensive in early disease stages and decreases over time. Brain. 2002;125:2202-12.

7. Liu JS, Zhao ML, Brosnan CF, Lee SC. Expression of inducible nitric oxide synthase and nitrotyrosine in multiple sclerosis lesions. Am J Pathol. 2001;158:2057-66.

8. Smith $\mathrm{KJ}$, Lassmann $\mathrm{H}$. The role of nitric oxide in multiple sclerosis. Lancet Neurol. 2002;1:232-41.

9. Lassmann H. Multiple sclerosis: lessons from molecular neuropathology. Exp Neurol. 2014;262PA:2-7.

10. Waxman SG. Axonal conduction and injury in multiple sclerosis: the role of sodium channels. Nat Rev Neurosci. 2006;7:932-41.

11. Nave K-AA, Trapp BD. Axon-glial signaling and the glial support of axon function. Annu Rev Neurosci. 2008;31:535-61.
12. Simons M, Misgeld T, Kerschensteiner M. A unified cell biological perspective on axon-myelin injury. J Cell Biol. 2014;206:335-45.

13. Nikic I, Merkler D, Sorbara C, Brinkoetter M, Kreutzfeldt M, Bareyre FM, et al. A reversible form of axon damage in experimental autoimmune encephalomyelitis and multiple sclerosis. Nat Med. 2011;17:495-9.

14. Ghafourifar P, Mousavizadeh K, Parihar MS, Nazarewicz RR, Parihar A, Zenebe WJ. Mitochondria in multiple sclerosis. Front Biosci. 2008;13:3116-26.

15. Campbell GR, Worrall JT, Mahad DJ. The central role of mitochondria in axonal degeneration in multiple sclerosis. Mult Scler. 2014;20:1806-13.

16. Witte ME, Mahad DJ, Lassmann H, van Horssen J. Mitochondrial dysfunction contributes to neurodegeneration in multiple sclerosis. Trends Mol Med. 2014;20:179-87.

17. Mahad DJ, Ziabreva I, Lassmann H, Turnbull D. Mitochondrial defects in acute multiple sclerosis lesions. Brain. 2008;131:1722-35.

18. Dutta R, McDonough J, Yin X, Peterson J, Chang A, Torres T, et al. Mitochondrial dysfunction as a cause of axonal degeneration in multiple sclerosis patients. Ann Neurol. 2006;59:478-89.

19. Sorbara CD, Wagner NE, Ladwig A, Nikic I, Merkler D, Kleele T, et al. Pervasive axonal transport deficits in multiple sclerosis models. Neuron. 2014;84:1183-90.

20. Di Penta A, Moreno B, Reix S, Fernandez-Diez B, Villanueva M, Errea O, et al. Oxidative stress and proinflammatory cytokines contribute to demyelination and axonal damage in a cerebellar culture model of neuroinflammation. PloS ONE. 2013;8:e54722.

21. Dickey AS, Strack S. PKA/AKAP1 and PP2A/Bbeta2 regulate neuronal morphogenesis via Drp1 phosphorylation and mitochondrial bioenergetics. J Neurosci. 2011;31:15716-26.

22. Picard M, White K, Turnbull DM. Mitochondrial morphology, topology, and membrane interactions in skeletal muscle: a quantitative three-dimensional electron microscopy study. J App Physiol. 2013;114:161-71.

23. Kiryu-Seo S, Ohno N, Kidd GJ, Komuro H, Trapp BD. Demyelination increases axonal stationary mitochondrial size and the speed of axonal mitochondrial transport. J Neurosci. 2010;30:6658-66.

24. Kasri NN, Govek EE, Van Aelst L. Characterization of oligophrenin-1, a RhoGAP lost in patients affected with mental retardation: lentiviral injection in organotypic brain slice cultures. Methods Enzymol. 2008;439:255-66.

25. Ohno N, Kidd GJ, Mahad D, Kiryu-Seo S, Avishai A, Komuro H, et al. Myelination and axonal electrical activity modulate the distribution and motility of mitochondria at CNS nodes of Ranvier. J Neurosci. 2011;31:7249-58.

26. Gnaiger E, Kuznetsov AV, Schneeberger S, Seiler R, Brandacher G, Steurer W, et al. Mitochondria in the cold. In: Heldmaier $\mathrm{G}$ et al., editors. Life in the cold. Berlin Heidelberg: Springer; 2000. p. 431-42.

27. Mannella CA. Structure and dynamics of the mitochondrial inner membrane cristae. Biochim Biophys Acta. 2006;1763:542-8.

28. Cogliati S, Frezza C, Soriano ME, Varanita T, Quintana-Cabrera R, Corrado M, et al. Mitochondrial cristae shape determines respiratory chain supercomplexes assembly and respiratory efficiency. Cell. 2013;155:160-71.

29. Zhang CL, Ho PL, Kintner DB, Sun D, Chiu SY. Activity-dependent regulation of mitochondrial motility by calcium and Na/K-ATPase at nodes of Ranvier of myelinated nerves. J Neurosci. 2010;30:3555-66.

30. Chiu SY. Matching mitochondria to metabolic needs at nodes of Ranvier. Neuroscientist. 2011;17:343-50.

31. Fischer F, Hamann A, Osiewacz HD. Mitochondrial quality control: an integrated network of pathways. Trends Biochem Sci. 2012;37:284-92.

32. Hollenbeck PJ, Saxton WM. The axonal transport of mitochondria. J Cell Sci. 2005;118:5411-9.

33. Sheng ZH, Cai Q. Mitochondrial transport in neurons: impact on synaptic homeostasis and neurodegeneration. Nat Rev Neurosci. 2012;13:77-93.

34. Pathak D, Sepp KJ, Hollenbeck PJ. Evidence that myosin activity opposes microtubule-based axonal transport of mitochondria. J Neurosci. 2010;30:8984-92.

35. Lassmann $\mathrm{H}$, van Horssen J. The molecular basis of neurodegeneration in multiple sclerosis. FEBS Lett. 2011;585:3715-23.

36. Davies AL, Desai RA, Bloomfield PS, Mclntosh PR, Chapple KJ, Linington C, et al. Neurological deficits caused by tissue hypoxia in neuroinflammatory disease. Ann Neurol. 2013;74:815-25.

37. Lambert AJ, Brand MD. Reactive oxygen species production by mitochondria. Meth Mol Biol. 2009;554:165-81.

38. Turrens JF. Mitochondrial formation of reactive oxygen species. J Physiol. 2003;552:335-44. 
39. Venditti P, Di Stefano L, Di Mateo S. Mitochondrial metabolism of reactive oxygen species. Mitochondrion. 2013;13:71-82.

40. Morris RL, Hollenbeck PJ. The regulation of bidirectional mitochondrial transport is coordinated with axonal outgrowth. J Cell Sci. 1993;104 (Pt 3):917-27.

41. Ruthel G, Hollenbeck PJ. Response of mitochondrial traffic to axon determination and differential branch growth. J Neurosci. 2003;23:8618-24.

42. Waxman SG. Mechanisms of disease: sodium channels and neuroprotection in multiple sclerosis-current status. Nat Clin Pract Neurol. 2008;4:159-69.

43. Misgeld T, Kerschensteiner M, Bareyre FM, Burgess RW, Lichtman JW. Imaging axonal transport of mitochondria in vivo. Nat Methods. 2007:4:559-61.

44. Mahad DJ, Ziabreva I, Campbell G, Lax N, White K, Hanson PS, et al. Mitochondrial changes within axons in multiple sclerosis. Brain. 2009;132:1161-74

\section{Submit your next manuscript to BioMed Central and take full advantage of:}

- Convenient online submission

- Thorough peer review

- No space constraints or color figure charges

- Immediate publication on acceptance

- Inclusion in PubMed, CAS, Scopus and Google Scholar

- Research which is freely available for redistribution 